\title{
Demanda/Control y la Salud Mental en Profesionales de la Salud: Un Estudio en Seis Países Latinoamericanos.
}

\section{Demand/Control Model and Mental Health in Health Professionals: A Study in Six Latin-American Countries}

Fecha de recepción: 9/09/2014

Fecha de aceptación: 11/12/2014

\author{
Arturo Juárez García ${ }^{1}$, Aldo Vera Calzaretta ${ }^{2}$, \\ César Merino Soto ${ }^{3}$, Viviola Gómez Ortíz ${ }^{4}$, \\ Lya Feldman ${ }^{5}$ y Elena Hernández Mendoza 6 \\ ${ }^{1}$ CITPSI, Universidad Autónoma del Estado de Morelos, Cuernavaca México; \\ ²Escuela de Salud Pública, Universidad de Chile, Santiago-Chile; \\ ${ }^{3}$ Universidad de San Martín de Porres, Lima Perú; \\ ¿Universidad de los Andes, Bogotá Colombia; \\ 5Universidad Simón Bolívar, Caracas Venezuela; \\ EUniversidad Nacional de Tucumán, Tucumán Argentina.
}

\section{resumen/alsstract:}

El Modelo Demanda/Control, mediante el cuestionario del contenido del trabajo (JCO) es quizás el método más estudiado para explorar las condiciones psicosociales del trabajo relacionadas a los problemas de salud en la investigación. A pesar de que es utilizado extensamente en Norteamérica y Europa, en Latinoamérica su uso es escaso. De esta forma, como parte de un estudio multicéntrico, se concentraron datos ( $N=989)$ de seis países latinoamericanos (Argentina, Chile, Colombia, México, Perú y Venezuela) en los que se analizó la asociación entre las variables del modelo y la salud mental en profesionales de la salud. Los resultados mostraron que las demandas psicológicas, el control y apoyo social estuvieron relacionados con la salud mental en todas las muestras controlando por factores demográficos, sin embargo los mejores predictores fueron diferentes dependiendo del país. El control fue el mejor predictor para Argentina (contribución relativa a $\mathrm{R}$-CRR- 68.7), demandas para México (CRR 83.3) y Perú (CRR 27.6); y para Chile, Colombia y Venezuela, el apoyo social parece ser el más importante (CRR=17.7, 41.9, 57.9 respectivamente). La relación universal de las variables del modelo Demanda/Control y la Salud Mental fue confirmada en este estudio. Se sugieren futuros estudios psicométricos del JCQ que exploren su funcionamiento diferencial en diferentes culturas latinas.

The Demand/Control Model measured utilizing the Job content questionnaire (JCO) is perhaps the most widely used model for exploring psychosocial work conditions and their relationship to health problems. While this model is widely used in North-America and Europe, in Latin-America knowledge of the model remains almost unknown. This study utilizes data from ( $N=989$ ) health workers in six Latin-America countries (Argentine, Chile, Colombia, México, Peru and Venezuela) in which the association between variables of the Demand/Control Model and mental health disorders utilizing GHO questionnaire in health workers is analyzed. The results show that psychological demands, control, and social support are all related to mental health controlling for demographical factors in all of the countries. However the best predictors were different depending on the country; thus control was the most important for Argentina (CRR 68.7), demands for México (CRR 83.3) and Perú (CRR 27.6), and social support appeared to be the most important for Chile, Colombia and Venezuela (CRR= 17.7, 41.9, 57.9 respectively). The important relationship between Demand/Control variables and mental health is supported in this study. Further psychometric analyses of the JCO exploring differential item functioning of the scales in different Latin-American cultures are suggested. 


\section{palabras clave/keywords:}

Demanda/Control, JCQ, Salud mental, GHQ, Latinoamérica.

\section{Introducción.}

En los últimos 40 años, en medio de las grandes transformaciones de las condiciones de trabajo y los mercados laborales, la emergencia de modelos explicativos de los procesos psicosociales ligados al estrés laboral y sus efectos en la salud ha sido notoria. El modelo con mayor trascendencia por sus evidencias empíricas y su difusión es definitivamente el modelo demanda/control o modelo de tensión laboral (job strain por sus siglas en ingles) de Karasek (1976). La hipótesis medular del mismo es que no solo las demandas psicológicas que el trabajo impone definen la experiencia del estrés y el aumento de riesgo para la enfermedad, sino que también es fundamental el grado de control que los trabajadores tengan sobre su tarea. De esta manera se postula un mecanismo interactivo y de sinergia entre ambas variables o un proceso a través del cual el control modera los efectos de las demandas (Der Doef and Maes, 1990).

Las demandas psicológicas en general hacen referencia a la carga de trabajo percibida, incluyendo la cantidad de trabajo, el ritmo, la presión de atención, los límites de tiempo y las interrupciones entre las principales exigencias. Con respecto al control, éste se refiere más bien al contenido del trabajo e incorpora dos dimensiones: la autonomía (libertad de decisión $)^{1}$ y la utilización de habilidades. La primera representa el margen que tiene la persona para decidir sobre su tarea o influir en la forma de hacerla y por tanto de controlarla; la segunda se refiere a la posibilidad que brinda el trabajo de que la persona utilice sus habilidades y su creatividad, así como la posibilidad de aprender en el mismo (Karasek, 1998).

Vislumbrando la combinación de ambas variables en la caracterización del trabajo, Karasek y Theorell (1990) propusieron 4 condiciones de riesgo psicosocial: 1) trabajos de tensión alta, 2) trabajos activos, 3) trabajos de poca tensión y 4) trabajos pasivos (Figura 1). La hipótesis de la tensión laboral se comprobó con datos de trabajadores Suecos y Norteamericanos cuyos diseños fueron transversales y longitudinales, quedando establecido desde entonces el potencial predictivo del modelo (Karasek y Theorell, 1990). No obstante, también ha recibido varias críticas (De Jonge y Kompier, 1997; Soderfeldt, Soderfeldt, Muntaner, O’Campo, Warg, y Ohlson, 1996).

Por su parte, Johnson (1986) argumentó como insuficiente abordar los fenómenos sociales del trabajo sólo con las demandas y el control, y consideró que una de las esferas altamente necesarias para el análisis psicosocial laboral y su influencia en la salud corresponde a las

\footnotetext{
${ }^{1}$ Algunas traducciones refieren al control como la "capacidad de decisión" en lugar de la "libertad de decisión" (decision latitude), sin embargo, el concepto refiere a una oportunidad que se encuentra en el entorno y las características de la tarea que permiten el uso de las capacidades del individuo, pero no a las capacidades del individuo en sí mismas.
}

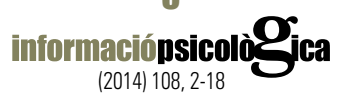




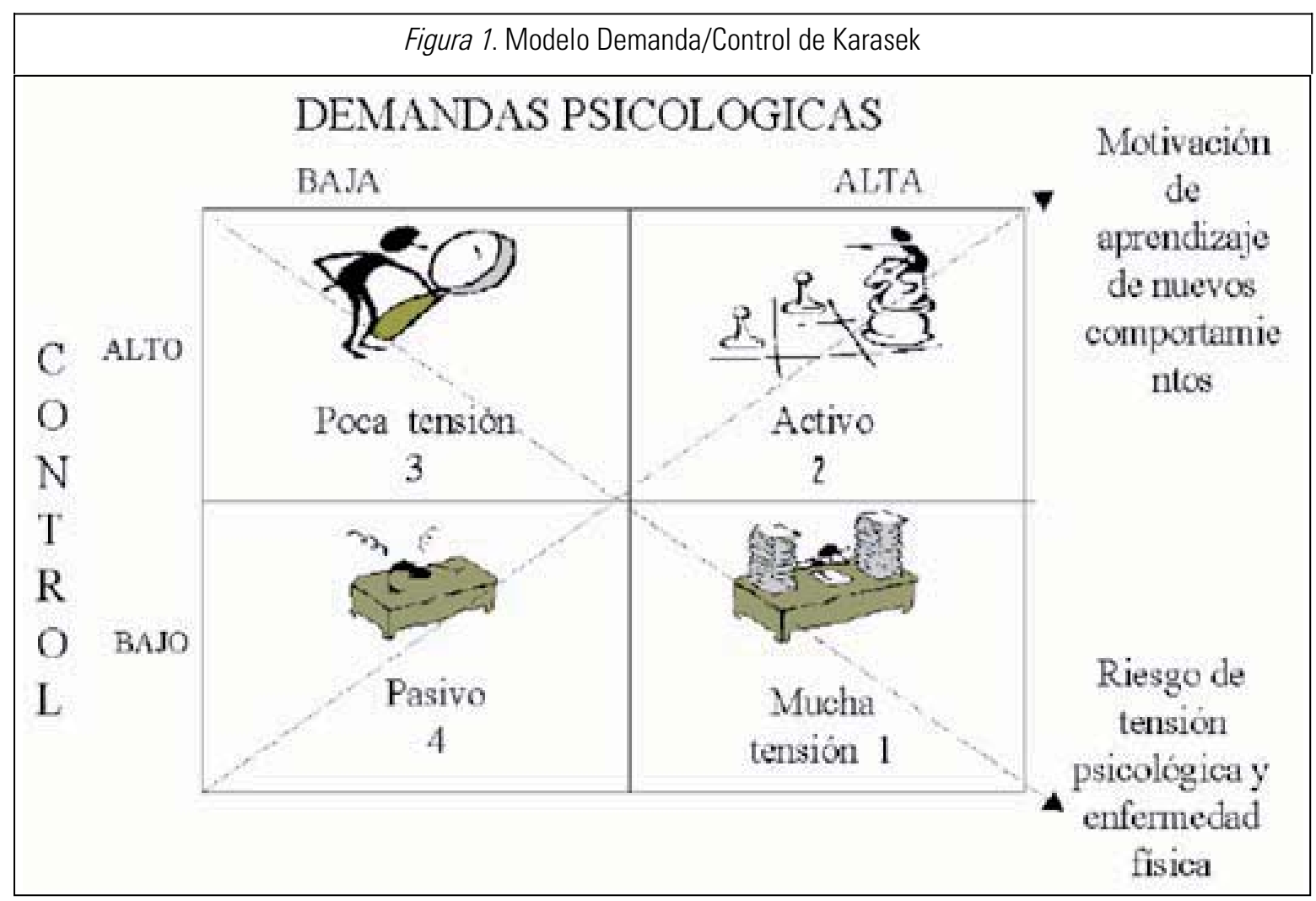

Fuente: Elaboración propia con base en el modelo original (Karasek y Theorell, 1990)

relaciones interpersonales dentro del medio laboral: el apoyo social. Por éste, Karasek (1998) se refiere a los niveles generales de ayuda que están disponibles en las interacciones sociales en el trabajo (compañeros de trabajo y supervisores). Los estudios demostraron que el apoyo social mejoraba las predicciones de nuevos casos de enfermedad cardiovascular de las hechas sólo por las demandas y el control (Johnson \& Hall, 1988; Schnall, Belkic, Landsbergis \& Baker, 2000). De este modo, el modelo se transforma en la versión demanda/control/apoyo (iso-strain en inglés), cuya traducción pudiera ser "tensión laboral en aislamiento", y aunque resulta complejo definir los mecanismos por los cuales la falta de apoyo social laboral afecta el bienestar de los trabajadores, en el modelo se considera un papel principalmente moderador (Karasek y Theorell, 1990).

Diversas investigaciones confirman la predicción del modelo en distintos resultados de salud en trabajadores, por citar algunas, se encuentran en relación con: desórdenes musculo-esqueléticos (Skov, Vilhelm, \& Orhede,1996; Engström, Hanse \& Kadefors 1999; Deveraux, 1999), ausencias por enfermedad (Niedhammer, Bugel, Goldberg, Marcel \& Guéguen,, 1998;), enfermedades cardiovasculares y riesgos coronarios (Schnall et. al, 2000; Kivimaki, Virtanen, Elovainio, Kouvonen, Vaananen \& Vahtera, ; 2006), trastornos de sueño (Theorell, Schüldt, Ekholm, \& Michélsen, 1995), diabetes (Nyberg, Fransson, Heikkila, Ahola, Alfredsson, Bjorner \& Kivimaki, 2014), enfermedades y salud en general (Petterson \& Arnetz, 1997; Akker, Buntinx, Metsemakers \& Knottnerus, 2000;), incluso la muerte (Michie \& Cockcroft, 1996). 
Definitivamente las variables de salud con las que el modelo tiene mayor asociación y extensa evidencia científica parecen corresponder a las mismas con las que iniciaron las investigaciones originalmente, específicamente a las enfermedades cardiovasculares y los desórdenes de salud mental (Karasek, 1979; Karasek y Theorell, 1990). En cuanto a la salud mental, las revisiones sistemáticas y los meta-análisis que concentran una vasta cantidad de estudios, confirman la afectación mental ante la sinergia de altas demandas y bajo control (Stansfeld y Candy, 2000; Der Doef y Maes, 1999).

No obstante este abundante cuerpo de evidencias en países desarrollados, la investigación del modelo de tensión laboral y su relación con la salud en regiones latinoamericanas sigue siendo magra, y su aplicación práctica y preventiva en los escenarios laborales sigue siendo ampliamente soslayada, lo que contrasta con condiciones de trabajo existentes que son más adversas que en países desarrollados (Rodríguez y Mendoza, 2007, OIT, 2012). En particular las organizaciones de servicio humano, aquellas cuyo objeto de trabajo son otras personas, son las más vulnerables al estrés y los factores psicosociales (Gil Monte \& Peiró, 1996). Diversos estudios en países desarrollados han demostrado la vulnerabilidad de los profesionales de la salud a las altas demandas y el bajo control, así como ante diversos desórdenes mentales y psiquiátricos (Bourbonnais, Comeau, \& Vezina, 1999; Hurrel, Murphy, Sauter y Levi, 1988; Sauter, Murphy, \& Hurrell, 1990; Estryn-Behar, Kaminski, Peigne, Bonnet, Vaichère, Gozlan, Azoulay \& Giorgi,., 1990).

Una revisión sobre el uso del modelo demanda/control en Latinoamérica hasta el 2010, encontró tan sólo 30 artículos de investigación, los que en su mayoría son con muestras brasileñas (Geco, Bitencourt, Magnago, Bosi de Souza, Prochnow, Beck, Colomé \& Tavares, 2011). Específicamente las investigaciones del modelo en relación con la salud mental en profesionales de la salud se ubicó en tan sólo 4 estudios latinoamericanos (Araujo, Aquino, Menezes, Oliveira \& Aguiar, 2003; Pinho y Araujo, 2007; Gómez, 2011; Urbanetto, Magalhaes, Maciel, Sant'Anna, Gustavo, Poli-de-Figueiredo \& Magnago, 2013). En todos ellos se comprueba la relación positiva entre las demandas y el bajo control con los desórdenes mentales; sin embargo, 3 de ellos se realizaron en Brasil y se desconoce si estos hallazgos pueden hacerse extensivos a otros países latinos de habla hispana.

Dado lo anterior, es de suma importancia identificar las afectaciones en la salud mental que puedan estar asociadas a las características psicosociales que propone el modelo demanda/ control en distintos países latinos. Este hallazgo brindará elementos para determinar la universalidad del modelo y la incorporación de elementos psicosociales en los programas de prevención y vigilancia epidemiológica de los programas de salud laboral latinoamericanos.

De manera concreta, el objetivo del presente trabajo es identificar la asociación entre las variables del modelo demanda/control y la salud mental en trabajadores de la salud en 6 países latinoamericanos: Argentina, Chile, Colombia, México, Perú y Venezuela.

\section{Método}

\section{Participantes}

Como parte de un estudio multicéntrico latinoamericano más amplio en el tema de factores psicosociales laborales, en el presente trabajo se analiza la relación entre las variables del 
modelo demanda/control/apoyo social y la salud mental en un estudio con diseño observacional, transeccional/transversal.

Participaron un total de 989 empleado(a)s de los seis países, de los cuales 255 son mexicano(a)s, 65 Chileno(a)s y 285 colombiano(a)s, 173 peruano(a)s, 104 argentino(a)s, y 107 venezolano(a)s. Se tratan de profesionales de servicios de salud (enfermeras, médicos, laboratoristas, cuidadores, psicólogos, etc.) que fueron elegidos de forma no aleatoria y por conveniencia en diversos centros de trabajo (Tabla 1).

\begin{tabular}{|c|c|c|c|c|c|c|c|c|c|c|c|c|c|c|}
\hline \multicolumn{15}{|c|}{ Tabla 1. Distribución de las características demográficas de los grupos multinacionales } \\
\hline & \multicolumn{2}{|c|}{$\begin{array}{l}\text { México } \\
(n=255)\end{array}$} & \multicolumn{2}{|c|}{$\begin{array}{c}\text { Chile } \\
(\mathrm{n}=65)\end{array}$} & \multicolumn{2}{|c|}{$\begin{array}{c}\text { Perú } \\
(\mathrm{n}=173)\end{array}$} & \multicolumn{2}{|c|}{$\begin{array}{l}\text { Argentina } \\
(n=104)\end{array}$} & \multicolumn{2}{|c|}{$\begin{array}{l}\text { Colombia } \\
(n=285)\end{array}$} & \multicolumn{2}{|c|}{$\begin{array}{c}\text { Venezuela } \\
(n=107)\end{array}$} & \multicolumn{2}{|c|}{$\begin{array}{l}\text { Total } \\
\text { (989) } \\
\end{array}$} \\
\hline & $\mathrm{n}$ & $\%$ & $\mathrm{n}$ & $\%$ & $\mathrm{n}$ & $\%$ & $n$ & $\%$ & $n$ & $\%$ & $\mathrm{n}$ & $\%$ & n & $\%$ \\
\hline \multicolumn{15}{|l|}{ Sexo } \\
\hline Masculino & 45 & 18.8 & 5 & 7.7 & 75 & 43.4 & 26 & 25.5 & 32 & 11.3 & 49 & 45.8 & 232 & 23.9 \\
\hline Femenino & 195 & 81.3 & 60 & 92.3 & 98 & 56.6 & 76 & 74.5 & 252 & 88.7 & 58 & 54.2 & 739 & 76.1 \\
\hline \multicolumn{15}{|l|}{ Profesión } \\
\hline Enfermero(a) & 206 & 80.8 & 65 & & 30 & 17.3 & 104 & & 285 & & 0 & .0 & 690 & 69.8 \\
\hline Médico & 49 & 19.2 & 0 & .0 & 74 & 42.8 & 0 & .0 & 0 & .0 & 0 & .0 & 123 & 12.4 \\
\hline Varios & 0 & .0 & 0 & .0 & 69 & 39.9 & 0 & 0 & 0 & .0 & 107 & 100.0 & 176 & 17.8 \\
\hline \multicolumn{15}{|l|}{ Estado civil } \\
\hline Sin pareja & 87 & 34.4 & 23 & 36.5 & 57 & 32.9 & 47 & 45.2 & 0 & .0 & 50 & 46.7 & 264 & 37.7 \\
\hline \multirow[t]{2}{*}{ Con pareja } & 166 & 65.6 & 40 & 63.5 & 116 & 67.1 & 57 & 54.8 & 0 & .0 & 57 & 53.3 & 436 & 62.3 \\
\hline & $\underline{M}$ & $\underline{\mathrm{DE}}$ & $\underline{M}$ & $\underline{\mathrm{DE}}$ & $\underline{M}$ & $\underline{\mathrm{DE}}$ & $\underline{M}$ & $\underline{\mathrm{DE}}$ & $\underline{M}$ & $\underline{\mathrm{DE}}$ & $\underline{\mathrm{M}}$ & $\underline{\mathrm{DE}}$ & $\underline{M}$ & $\underline{\mathrm{DE}}$ \\
\hline Edad & 39.30 & 9.81 & 47.86 & & 43.06 & 8.31 & 41.05 & 9.09 & 36.91 & 9.78 & 38.77 & 11.00 & 39.98 & 10.16 \\
\hline
\end{tabular}

\section{Instrumentos}

Se utilizó el Cuestionario del Contenido del Trabajo (JCQ por sus siglas en inglés) (Karasek, Brisson, Kawakami, Houtman, Bongers, \& Amick, 1998) en su versión 1.0 de 22 ítems que comprende las escalas de demandas psicológicas (e.g "tengo que trabajar muy rápido") con 6 ítems, control laboral (autonomía y utilización de habilidades) con 9 ítems (e.g "tengo mucha libertad para decidir cómo hacer mi trabajo" ó "para mi trabajo necesito ser creativo"), apoyo social del jefe con 4 ítems (e.g "mi jefe ayuda a que el trabajo se realice") y apoyo social de los compañeros con 4 ítems (e.g "mis compañeros de trabajo son amigables"), todas ellas con 4 opciones de respuesta que van desde "totalmente en desacuerdo" hasta "totalmente de acuerdo".

Se aplicó también el Cuestionario General de Salud (GHQ por sus siglas en inglés) (Goldberg, Gater, Sartorious, Ustun, Piccinelli, Gureje, \& Rutter, 1997) que ofrece indicadores 
de desórdenes psicológicos o afectaciones a la salud mental que se han clasificado como: síntomas somáticos, depresión, problemas de sueño/ansiedad y disfunción social. En esta versión (28 ítems, cada escala con 7 ítems) existe una opción de valoración total de desórdenes psicológicos hasta por un máximo de 28 síntomas, en donde a mayor puntuación o síntomas, mayores problemas de salud mental. Para el presente estudio, se usó la puntuación total considerando que la estructura multidimensional del GHQ-28 parece ser aún inestable en estudios multinacionales (Prady, Miles, Pickett, Fairley, Bloor, Gilbody, Kiernan, Mann \& Wright, 2013; Werneke, Goldberg, Yalcin \& Üstun, 2000).

\section{Análisis de Datos}

Se analizaron estadísticos descriptivos para cada una de las puntuaciones de los cuestionarios GHQ-28 y JCQ. Para examinar las relaciones entre ambas mediciones el enfoque se basó en la influencia única de los constructos del JCQ sobre los desórdenes psicológicos evaluados por el GHQ-28. Se usó la regresión lineal múltiple, obteniendo los estadísticos usuales (coeficientes B y beta) e indicadores de importancia relativa de las variables explicativas, es decir, coeficientes estructurales (Courville \& Thompson, 2001; Nimon, Zientek \& Henson, 2012) y pesos relativos (Johnson, 2000; Nimon et al., 2012), presentados en forma porcentual (Lorenzo-Seva, Ferrando \& Chico, 2010). Estos resultados se obtuvieron por medio del programa de Lorenzo-Seva et al. (2010).

Para el modelamiento mediante este procedimiento, se hicieron algunos cálculos psicométricos y re-especificaciones con el fin de mejorar sus requerimientos. Primero, como se esperaban distribuciones con distorsiones en la asimetría respecto a la normalidad teórica en las puntuaciones del GHQ-28 (y plausiblemente, también en JCQ), se verificó si los residuales en los modelos evaluados tendrían también alejamientos severos de la normalidad. Ya que se hallaron estos sesgos, se aplicó la transformación $L n$ en las puntuaciones del GHQ-28 y JCQ; previamente, se añadió una constante (1) a todas las puntuaciones del GHQ para eliminar las puntuaciones cero, una de las condiciones para la efectiva transformación $L n$. Segundo, ya que las diferencias en la fiabilidad ${ }^{2}$ de las puntuaciones añadirían diferentes montos de precisión a los resultados (Nimon et al. 2012; Thompson \& Vacha-Haase, 2000), se desatenuaron las correlaciones mediante la formulación de Spearman (1904). Estas correlaciones usaron la fiabilidad por consistencia interna estimada de las variables explicativas (puntuaciones del JCQ) en cada país, tomando en cuenta que este parámetro psicométrico es una propiedad de la puntuación y las características estadísticas de la muestra y no es intrínseco al instrumento (Nimon et al. 2012; Thompson \& Vacha-Haase, 2000). El coeficiente de fiabilidad usado fue $a$ de Cronbach (Cronbach, 1951), luego de verificar informalmente que los ítems satisfacían moderadamente el modelo equivalente tau latente, aspecto estadístico para la correcta interpretación de este coeficiente (Cronbach, 1951; 2004).

\section{Resultados.}

Antes del análisis principal, se establecieron los indicadores a incorporar en los modelos de regresión de acuerdo con los correspondientes criterios estadísticos. Por ejemplo, algunas

\footnotetext{
${ }^{2}$ Siendo conceptos sinónimos, en Latinoamérica es más frecuente encontrar el concepto de "confiabilidad" en lugar de "fiabilidad", no obstante se utiliza este último en todo el texto dado que es más común su uso en el contexto español.
} 
puntuaciones fueron integrados en uno sólo para evitar problemas de colinealidad entre los predictores; específicamente, en primer lugar, la integración de la puntuación de segundo orden Control Laboral, que usualmente está compuesto por las variables de Autoridad de Decisión y Utilización de Habilidades (Karasek y Theorell, 1990; González, Juárez, Camacho, Noriega y Escobedo, 2014); en el presente estudio ambos correlacionaron 0.61 ( $p<$ 0.01), así que para el análisis de regresión se utilizó la puntuación Control Laboral incorporando ambas sub-dimensiones. En segundo lugar, esto también se aplicó al área de Apoyo Social Total, correspondiente a las puntuaciones de Apoyo de Compañeros y Apoyo del Jefe $(r=0.51, p<0.01)$.

Debido a que el impacto del poder discriminativo de los ítems influencia directamente la fiabilidad y validez interna de las puntuaciones (Nunnally \& Bernstein, 1995), se verificó si los ítems mantenían razonables propiedades psicométricas en cada país, tratando de remover la mínima cantidad de ítems con pobres propiedades consistentes (Nimon et al., 2012). Los ítems de GHQ-28 funcionaron bien o moderadamente bien en cada país; para el JCQ, en el caso de la escala Demandas Psicológicas, no se incluyó en su cálculo los ítems 14 ("tengo suficiente tiempo para terminar mi trabajo") y 16 ("En mi trabajo tengo que responder a órdenes contradictorias") debido a que consistentemente mostraron bajas correlaciones ítem-test en cada país. La desatenuación de las correlaciones y estos ajustes realizados en algunas de las subescalas permitiría un control a posteriori del error de medición sobre las estimaciones en el modelo de regresión (Johnson, 2004; Nimon, et al., 2012). Finalmente, no se detectaron puntuaciones que puedan identificarse como casos extremos genuinos en los datos del GHQ-28, aun cuando las distribuciones fueron altamente sesgadas (especialmente GHQ-28), pues debido al constructo medido por el instrumento, estos deben ser inherentes a la muestra de estudio (problemas importantes de salud mental en algunos casos). Esta situación también se aplicó al JCQ.

Las características demográficas respecto a la profesión variaron entre países (Tabla 1), excepto el estado civil que fue comparativamente más homogéneo y el sexo, en donde el femenino fue más frecuente en algunas muestras. Dadas las diferencias distribucionales, el sexo y el estado civil se ingresaron en el modelo de regresión para aislar su contribución única sobre el resto de variables (escalas del JCQ). Las propiedades descriptivas y de fiabilidad de las puntuaciones mostradas indican que, para el GHQ-28 (Tabla 2), ocurren distribuciones con fuerte asimetría positiva y con excesos de curtosis, resultado que podría esperarse debido a la naturaleza del constructo en población presumiblemente normal. La excepción son Chile y Perú, que mostraron una mayor densidad de sus puntuaciones sobre sus medias, sugiriendo una mayor frecuencia de problemas de salud mental. Con algún riesgo metodológico por la imposibilidad de comparaciones directas, podemos inferir que de acuerdo a los 6 síntomas como punto de corte sugerido para determinar "casos" (MedinaMora, Padilla, Campillo, Campillo, Caraveo \& Corona, 1983), las medias de las muestras de Chile, Perú y Colombia dejan ver que se encuentran en riesgo a su salud mental. Con respecto a la consistencia interna del GHQ-28, este fue mayor a $.80 \mathrm{y}$ en algunos grupos mayor a.90, indicando elevada consistencia de respuestas.

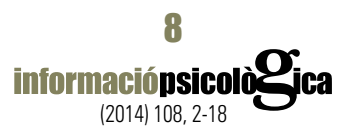




\begin{tabular}{|lcccc|}
\hline \multicolumn{5}{|c|}{ Tabla 2. Información descriptiva de las puntuaciones de GHQ-28 } \\
\hline M (DE) & As & Cu & $\alpha$ \\
\hline México & $3(5)$ & 5.0 & 2.3 & 0.93 \\
Chile & $8.2(7.7)$ & -1.0 & 0.5 & 0.94 \\
Perú & $6.3(4.8)$ & -1.1 & 0.2 & 0.83 \\
Argentina & $2.3(4.3)$ & 8.0 & 2.6 & 0.91 \\
Colombia & $7.3(4.1)$ & 2.6 & 1.7 & 0.80 \\
Venezuela & $3.1(5.9)$ & 5.3 & 2.5 & 0.96 \\
Total & $5.1(5.4)$ & 1.3 & 1.3 & 0.89 \\
\hline As: asimetría de Fisher; $C u$ : curtosis de Fisher; $\alpha$ : coeficiente de fiabilidad. & & \\
\hline
\end{tabular}

Con respecto a las variables del JCQ (Tabla 3), las puntuaciones más confiables y de magnitudes más parecidas entre los grupos fueron Control y Apoyo Total; éste último con menor error de medición en sus puntuaciones. Demandas Psicológicas fue muy variable en la fiabilidad, llegando a fiabilidades buenas (.83) y otras bajas (.32). Aunque no puede haber certeza en la viabilidad de las comparaciones directas, podemos notar que las medias de Demandas Psicológicas son más bajas que las reportadas en otras encuestas representativas en países desarrollados, mientras que el control y el apoyo social son más o menos similares (Karasek, et. al, 1998). En el mismo tenor y dentro de las limitaciones de comparabilidad directa, es inevitable observar que las medias mostraron a Venezuela como el país con las menores demandas, mayor control y mayor apoyo social, mientras que Perú fue el de menor apoyo y menor control (este último junto con Chile), y finalmente Colombia destacó con las mayores demandas psicológicas.

Para verificar el efecto sobre la salud mental de la combinación de demandas y control, primero se calculó el coeficiente de tensión laboral (que permite utilizarlo como una variable continua) de acuerdo la fórmula: "demandas psicológicas*2/control”, como se ha sugerido antes (Landsbergis, Schnall, Warren, Pickering, \& Schwartz, 1994). Después se analizó su relación con la salud mental en una estimación por correlación simple $\left(\mathrm{r}_{\mathrm{s}}\right)$. No fue factible incorporar este coeficiente en el modelo de regresión por los problemas de multicolinealidad esperados, así como la imposibilidad de obtener una fiabilidad del mismo, y por ello, de aplicar el procedimiento de desatenuación. En general, los resultados bivariados evidenciaron que las correlaciones de mayor fuerza con la salud mental se dan con este coeficiente (entre $r_{s=} .16$ y $r_{s=.} 31$ ) en comparación con las variables demanda, control o apoyo por separado y en las direcciones esperadas (mayor tensión laboral, mayores afectaciones a la salud mental) ${ }^{3}$.

\footnotetext{
${ }^{3}$ No se muestra por razones de espacio, dando prioridad a los hallazgos de la regresión múltiple.
} 


\begin{tabular}{|c|c|c|c|c|c|c|c|}
\hline \multicolumn{8}{|c|}{ Tabla 3. Información descriptiva de las puntuaciones de JCO } \\
\hline & México & Chile & Perú & Argentina & Colombia & Venezuela & Total \\
\hline $\begin{array}{l}\text { Control } \\
M \text { (DE) }\end{array}$ & $74.7(10.2)$ & $64(12)$ & $64.2(12.8)$ & $71(8)$ & $70.4(9.8)$ & $82.2(12.8)$ & $71.3(12)$ \\
\hline As & -0.6 & -0.1 & 0.1 & 0.5 & 0 & -2.2 & -0.3 \\
\hline$C u$ & 0.6 & 0.6 & -1.3 & 0.7 & 0.1 & 4.4 & -0.3 \\
\hline$\alpha$ & 0.70 & 0.63 & 0.79 & 0.57 & 0.74 & 0.94 & 0.81 \\
\hline $\begin{array}{l}\text { Uso de Hab } \\
\text { M (DE) }\end{array}$ & $36.6(4.5)$ & $33.3(5.6)$ & $31.7(6.6)$ & $36.1(3.7)$ & $37.3(4.9)$ & $39(5.6)$ & $35.9(5.7)$ \\
\hline As & -0.4 & -0.4 & -0.2 & 0.2 & -1.0 & -2.3 & -0.9 \\
\hline $\mathrm{Cu}$ & 0.0 & 0.3 & -0.8 & -0.1 & 2.3 & 5.0 & 0.7 \\
\hline$\alpha$ & 0.52 & 0.46 & 0.71 & 0.36 & 0.75 & 0.92 & 0.75 \\
\hline $\begin{array}{l}\text { Aut. Dec. } \\
\text { M (DE) }\end{array}$ & $38.1(7.4)$ & 30.7 (8.8) & $32.5(7.6)$ & $34.9(5.9)$ & $33.1(6.5)$ & $43.2(7.8)$ & 35.4 (8) \\
\hline As & -0.5 & -0.3 & 0.4 & 0.4 & 0.4 & -2 & -0.1 \\
\hline $\mathrm{Cu}$ & 0.3 & -0.1 & -1.1 & 0.1 & 0.1 & 3.7 & -0.5 \\
\hline$\alpha$ & 0.76 & 0.71 & 0.79 & 0.55 & 0.33 & 0.85 & 0.71 \\
\hline $\begin{array}{l}\text { Dem. Psic. } \\
\text { M (DE) }\end{array}$ & $20.7(6.9)$ & 21.7 (5.8) & $20.9(4)$ & $20.2(4.3)$ & $22(3.9)$ & $19.1(4.2)$ & $20.9(5.1)$ \\
\hline As & -0.1 & 0.1 & -0.2 & -0.4 & -0.1 & 0.3 & -0.1 \\
\hline $\mathrm{Cu}$ & -0.7 & -0.7 & 0.6 & 0.8 & 0.5 & -0.3 & 0.2 \\
\hline$\alpha$ & 0.83 & 0.78 & 0.49 & 0.45 & 0.32 & 0.67 & 0.30 \\
\hline $\begin{array}{l}\text { Apoyo. Total } \\
\text { M (DE) }\end{array}$ & $23.5(4.5)$ & $23.8(4.9)$ & $19.6(5)$ & $23.2(3.9)$ & 22.9 (3.5) & $26.5(6.5)$ & $23(4.9)$ \\
\hline As & -0.2 & -0.4 & -0.8 & -0.6 & -0.4 & -0.8 & -0.4 \\
\hline$C u$ & 0.2 & 0 & 0.2 & 1.3 & 0.8 & -0.7 & 0.5 \\
\hline$\alpha$ & 0.85 & 0.85 & 0.86 & 0.86 & 0.73 & 0.91 & 0.86 \\
\hline$M(D E)$ & $12.4(2.3)$ & $12.8(2.8)$ & $9.9(3)$ & $12.2(2.2)$ & $12(2)$ & $13.4(3.3)$ & $12(2.7)$ \\
\hline As & -0.3 & -0.7 & -0.7 & -0.7 & -0.3 & -1.1 & -0.7 \\
\hline $\mathrm{Cu}$ & 0.1 & 0.2 & -0.1 & 2.4 & 0.3 & 0 & 0.8 \\
\hline$\alpha$ & 0.82 & 0.89 & 0.88 & 0.85 & 0.69 & 0.83 & 0.84 \\
\hline $\begin{array}{l}\text { A. Jefe } \\
\text { M (DE) }\end{array}$ & $11(3.1)$ & $11(3.2)$ & $9.7(2.9)$ & $11(2.2)$ & $10.9(2.3)$ & $13.1(3.6)$ & $11(3)$ \\
\hline$A s$ & -0.5 & -0.3 & -0.5 & 0 & -0.5 & -1.0 & -0.3 \\
\hline$C u$ & -0.1 & -0.4 & -0.2 & 0.3 & 0.8 & -0.4 & -0.1 \\
\hline$\alpha$ & 0.89 & 0.88 & 0.82 & 0.80 & 0.70 & 0.88 & 0.83 \\
\hline $\begin{array}{l}\text { Tensión Lab. } \\
\text { M (DE) }\end{array}$ & $0.6(0.2)$ & $0.7(0.3)$ & $0.7(0.2)$ & $0.6(0.1)$ & $0.6(0.1)$ & $0.5(0.2)$ & $0.6(0.2)$ \\
\hline As & 0.7 & 1.1 & 0.1 & -0.3 & 0.4 & 3.0 & 0.9 \\
\hline $\mathrm{Cu}$ & 1.5 & 1.6 & -0.5 & 0.4 & 0.4 & 10.3 & 2.5 \\
\hline
\end{tabular}




\section{Regresión lineal}

En la Tabla 4 se muestran los estadísticos de ajuste, así como los coeficientes de regresión. Las estimaciones por medio de intervalos de confianza no pudieron obtenerse para Chile, Venezuela y Colombia por problemas de convergencia en el procedimiento bootstrap relacionados posiblemente con un fuerte desequilibrio en las frecuencias en alguno de los predictores o poca variabilidad, así que únicamente se presentarán los valores puntuales cuya información descriptiva aún es útil. Se obtuvo un ajuste similar y alrededor del $20 \%$ de la varianza o más de los desórdenes psicológicos fue explicada por los modelos en el caso de México, Chile y Perú; en contraste, en Argentina fue bajo y en Colombia prácticamente ocurrió como valor predictivo de cero. En éste último país, también puede observarse que las variables explicativas del presente no tuvieron un impacto sistemático que pueda valorarse, pues los coeficientes de regresión fueron todos cercanos a cero. En Venezuela se obtuvo un inusual alto grado de ajuste (más del $80 \%$ ), en que Control y Apoyo Total contribuyeron fuertemente a explicar la variabilidad de los desórdenes psicológicos medidos mediante el GHQ.

\begin{tabular}{|c|c|c|c|c|c|c|}
\hline \multicolumn{7}{|c|}{$\begin{array}{l}\text { Tabla 4. Efectos de variables demográficas y del modelo demanda/control (JCO) } \\
\text { sobre los desórdenes psicológicos (GHO) }\end{array}$} \\
\hline & $\begin{array}{c}\text { México } \\
(\mathrm{n}=231)\end{array}$ & $\begin{array}{l}\text { Chile } \\
(n=65)\end{array}$ & $\begin{array}{c}\text { Peru } \\
(n=173)\end{array}$ & $\begin{array}{l}\text { Argentina } \\
(n=102)\end{array}$ & $\begin{array}{l}\text { Colombia } \\
\text { (n=279) }\end{array}$ & $\begin{array}{l}\text { Venezuela } \\
(\mathrm{n}=107)\end{array}$ \\
\hline \multicolumn{7}{|l|}{ Ajuste del modelo } \\
\hline $\mathrm{R}$ & $\begin{array}{c}0.52 \\
(0.42 ; 0.70)\end{array}$ & 0.45 & $\begin{array}{c}0.48 \\
(0.408 ; 0.663)\end{array}$ & $\begin{array}{c}0.35 \\
(0.25 ; 0.61)\end{array}$ & 0.15 & 0.93 \\
\hline R2 & $\begin{array}{c}0.27 \\
(0.17 ; 0.50)\end{array}$ & 0.20 & $\begin{array}{c}0.23 \\
(0.166 ; 0.440)\end{array}$ & $\begin{array}{c}0.12 \\
(0.06 ; 0.38)\end{array}$ & 0.023 & 0.87 \\
\hline Coeficientes beta & & & & & & \\
\hline & $\begin{array}{c}\text { Beta } \\
\text { (inf.; sup.) }\end{array}$ & Beta & $\begin{array}{c}\text { Beta } \\
\text { (inf.; sup.) }\end{array}$ & $\begin{array}{c}\text { Beta } \\
\text { (inf.; sup.) }\end{array}$ & Beta & Beta \\
\hline Edad & $\begin{array}{c}-0.141 \\
(-0.32 ; 0.048)\end{array}$ & -0.24 & $\begin{array}{c}-0.064 \\
(-0.211 ; 0.105)\end{array}$ & $\begin{array}{c}0.06 \\
(-0.131 ; 0.282)\end{array}$ & 0.08 & 0.035 \\
\hline Sexo (varón = 1) & $\begin{array}{c}-0.033 \\
(-0.216 ; 0.118)\end{array}$ & -0.22 & $\begin{array}{c}0.162 \\
(-0.036 ; 0.411)\end{array}$ & $\begin{array}{c}-0.07 \\
(-0.256 ; 0.136)\end{array}$ & -0.04 & 0.67 \\
\hline $\begin{array}{l}\text { E. Civil } \\
(\sin \text { pareja }=1)\end{array}$ & $\begin{array}{c}0.087 \\
(-0.067 ; 0.277)\end{array}$ & -0.21 & $\begin{array}{c}0.257 \\
(0.08 ; 0.453)\end{array}$ & $\begin{array}{c}-0.11 \\
(-0.316 ; 0.075)\end{array}$ & - & 0.08 \\
\hline Control & $\begin{array}{c}0.02 \\
(-0.15 ; 0.278)\end{array}$ & -0.07 & $\begin{array}{c}-0.115 \\
(-0.553 ; 0.255)\end{array}$ & $\begin{array}{c}-0.31 \\
(-0.628 ; 0.01)\end{array}$ & 0.05 & -0.50 \\
\hline Dem. Psic. & $\begin{array}{c}0.489 \\
(0.352 ; 0.725)\end{array}$ & 0.04 & $\begin{array}{c}0.258 \\
(-0.041 ; 0.61)\end{array}$ & $\begin{array}{c}-0.02 \\
(-0.439 ; 0.273)\end{array}$ & 0.03 & -0.27 \\
\hline Apoyo total & $\begin{array}{c}-0.048 \\
(-0.293 ; 0.203)\end{array}$ & -0.23 & $\begin{array}{c}-0.263 \\
(-0.655 ; 0.149)\end{array}$ & $\begin{array}{c}-0.04 \\
(-0.377 ; 0.271)\end{array}$ & -0.10 & -0.67 \\
\hline
\end{tabular}




\begin{tabular}{|c|c|c|c|c|c|c|}
\hline \multicolumn{7}{|c|}{$\begin{array}{l}\text { Tabla 5. Importancia relativa de variables demográficas y del modelo demanda/control (JCQ) } \\
\text { sobre desórdenes psicológicos (GHO) }\end{array}$} \\
\hline & $\begin{array}{l}\text { México } \\
(\mathrm{n}=231)\end{array}$ & $\begin{array}{c}\text { Chile } \\
(\mathrm{n}=65)\end{array}$ & $\begin{array}{c}\text { Peru } \\
(n=173)\end{array}$ & $\begin{array}{l}\text { Argentina } \\
(\mathrm{n}=102)\end{array}$ & $\begin{array}{c}\text { Colombia } \\
\text { (279) }\end{array}$ & $\begin{array}{r}\text { Venezuela } \\
(\mathrm{n}=107)\end{array}$ \\
\hline \multicolumn{7}{|l|}{$\begin{array}{l}\text { Coeficientes } \\
\text { estructurales }\end{array}$} \\
\hline Edad & $\begin{array}{c}-0.33 \\
(-0.541 ; \\
-0.102)\end{array}$ & -0.58 & $\begin{array}{c}-0.19 \\
(-0.443 ; 0.101)\end{array}$ & $\begin{array}{c}0.068 \\
(-0.404 ; 0.46)\end{array}$ & 0.67 & 0.03 \\
\hline Sexo (varón = 1) & $\begin{array}{c}-0.00 \\
(-0.246 ; 0.208)\end{array}$ & -0.44 & $\begin{array}{c}0.422 \\
(0.126 ; 0.638)\end{array}$ & $\begin{array}{c}-0.35 \\
(-0.653 ; 0.18)\end{array}$ & -0.16 & 0.07 \\
\hline E. Civil (sin pareja=1) & $\begin{array}{c}0.16 \\
(-0.094 ; 0.409)\end{array}$ & -0.40 & $\begin{array}{c}0.249 \\
(-0.022 ; 0.456)\end{array}$ & $\begin{array}{c}-0.28 \\
(-0.657 ; 0.214)\end{array}$ & - & -0.01 \\
\hline Control & $\begin{array}{c}-0.26 \\
(-0.457 ;-0.022)\end{array}$ & -0.26 & $\begin{array}{c}-0.713 \\
(-0.892 ;-0.329)\end{array}$ & $\begin{array}{c}-0.87 \\
(-0.948 ;-0.308)\end{array}$ & 0.19 & -0.80 \\
\hline Dem. Psic. & $\begin{array}{c}0.93 \\
(0.737 ; 0.972)\end{array}$ & 0.38 & $\begin{array}{c}0.599 \\
(0.218 ; 0.795)\end{array}$ & $\begin{array}{c}0.02 \\
(-0.557 ; 0.47)\end{array}$ & 0.26 & 0.36 \\
\hline Apoyo total & $\begin{array}{c}-0.27 \\
(-0.513 ;-0.045)\end{array}$ & -0.37 & $\begin{array}{c}-0.383 \\
(-0.545 ;-0.167)\end{array}$ & $\begin{array}{c}-0.51 \\
(-0.835 ; 0.221)\end{array}$ & -0.62 & -0.92 \\
\hline $\begin{array}{l}\text { Contribución } \\
\text { relativa a R }\end{array}$ & $\%$ CRR & $\%$ CRR & $\%$ CRR & $\%$ CRR & $\%$ CRR & $\%$ CRR \\
\hline Edad & $\begin{array}{c}8.7 \\
(1.1 ; 25.1)\end{array}$ & 29.6 & $\begin{array}{c}3.4 \\
(0.4 ; 16.3)\end{array}$ & $\begin{array}{c}1.9 \\
(0.3 ; 27.8)\end{array}$ & 39.4 & 0.2 \\
\hline Sexo (varón = 1) & $\begin{array}{c}0.3 \\
(0 ; 8.1)\end{array}$ & 21.5 & $\begin{array}{c}12.8 \\
(3.9 ; 30.6)\end{array}$ & $\begin{array}{c}8.3 \\
(0.3 ; 35.6)\end{array}$ & 4.8 & 0.6 \\
\hline E. Civil (sin pareja=1) & $\begin{array}{c}2.5 \\
(0.1 ; 15.3)\end{array}$ & 18.8 & $\begin{array}{c}14.2 \\
(2.1 ; 30.9)\end{array}$ & $\begin{array}{c}8.4 \\
(0.5 ; 41.3)\end{array}$ & $-*$ & 0.2 \\
\hline Control & $\begin{array}{c}2.0 \\
(0.3 ; 10.8)\end{array}$ & 5.3 & $\begin{array}{c}24.4 \\
(4.2 ; 54.3)\end{array}$ & $\begin{array}{c}68.7 \\
(7.3 ; 80.8)\end{array}$ & 8.0 & 35.4 \\
\hline Dem. Psic. & $\begin{array}{c}83.3 \\
(55.7 ; 88.8)\end{array}$ & 7.0 & $\begin{array}{c}27.6 \\
(3.7 ; 54.3)\end{array}$ & $\begin{array}{c}0.4 \\
(0.4 ; 41.1)\end{array}$ & 5.8 & 5.7 \\
\hline Apoyo total & $\begin{array}{c}3.2 \\
(0.5 ; 15.8)\end{array}$ & 17.7 & $\begin{array}{c}17.6 \\
(6.3 ; 36.4)\end{array}$ & $\begin{array}{c}12.4 \\
(1.5 ; 53.7)\end{array}$ & 41.9 & 57.9 \\
\hline
\end{tabular}

Observando los coeficientes estandarizados de regresión para propósitos de comparabilidad (Tabla 4), se pueden identificar algunos patrones sobre la contribución única de los predictores. En todos los países, la edad, el sexo y el estado civil generalmente producen baja variabilidad dentro del modelo; la edad es consistente bajo y usualmente en el nivel del error de muestreo; algo similar ocurre con la influencia del sexo, en que además la dirección de su influencia se orienta hacia las mujeres (signo negativo de beta), es decir una 
pequeña tendencia a que las mujeres puntúen levemente mayores desórdenes psicológicos. La excepción ocurre en Venezuela, en que el impacto de esta variable es mayor comparado con los demás países, pero orientado hacia los varones. Los coeficiente estandarizados, sin embargo, no se relacionan directamente con la importancia que tienes sus variables en el contexto del ajuste del modelo; por lo tanto, se analizan los coeficientes de importancia.

Controlando el efecto de las variables demográficas anteriores, el efecto de Control (C), Demandas Psicológicas (DP) y Apoyo Total (AT) fue diferente no solo cuantitativamente, sino cualitativamente en algunas variables. Esto puede observarse mejor en los coeficientes de importancia de las variables explicativas presentadas en la Tabla 5. Excepto en Colombia con respecto a la edad ( $r$ estructural $>0.60)$, la importancia relativa de las variables demográficas generalmente es menor que las variables psicosociales laborales medidas por el JCQ. Además, la influencia demográfica es más errática dentro de cada país y entre los países. Por otro lado, la influencia de C, DP y AT tiende a ser sistemática (con excepción de Argentina, el resto de los intervalos de confianza no incluyen el cero) y teóricamente razonables, es decir, que AT y $\mathrm{C}$ generalmente se relacionan negativamente con los desórdenes mentales, mientras que DP lo hace positivamente, tal y como se esperaba teóricamente.

En la Tabla 5 se observa que DP muestra influencia positiva (en un sentido estadístico) sobre los desórdenes psicológicos, significando que el incremento en DP produce también un incremento de la experiencia de problemas de salud mental; sin embargo, este impacto es más fuerte en México y Perú ( $r=.93$ y .59 respectivamente). En Chile y Venezuela es moderadamente fuerte ( $\mathrm{r}=.38$ y .36 respectivamente) y bajo en Colombia ( $\mathrm{r}=.26)$. En Argentina, la importancia de esta variable no parece existir. Respecto a $\mathrm{C}$, su importancia es clara en Perú $(\mathrm{r}=-.71)$, Argentina $(\mathrm{r}=-.87)$ y Venezuela $(\mathrm{r}=-.80)$, mientras que el resto de países su importancia puede considerarse baja. Finalmente, AT su mayor influencia ocurre en los países de Colombia y Venezuela ( $\mathrm{r}=-.62$ y -.92 respectivamente).

En un análisis intra-país, la mayor influencia sobre los desórdenes psicológicos de los mexicanos es DP comparado con C y AT; en Chile, influencia más la disminución de AT, aunque es moderadamente baja. En Perú, el aumento de DP y la disminución de AT. En Argentina, el impacto del Control es abrumador sobre la salud mental en comparación con DP y AT; entre los colombianos y venezolanos, la disminución de AT parece ser más relevante en términos de su afectación mental.

\section{Discusión.}

En general, los hallazgos de esta investigación comprueban la relación universal existente entre las variables del modelo demanda/control/apoyo y la salud mental en profesionales de la salud latinoamericanos, lo que significa que tales variables debieran ser objeto de control y vigilancia permanente en estos centros de trabajo. Tanto los efectos directos de las variables demandas, control y apoyo, como los encontrados con el coeficiente de tensión laboral en la salud mental, confirman la hipótesis teórica del modelo y muestran la importancia de intervenir conjuntamente en esas tres variables dado su potencial sinérgico. De esta forma, un adecuado análisis y distribución de cargas de trabajo, el fomentar la participación y autonomía, impulsar el uso de las habilidades, y promover las fuentes de apoyo socio-emocional

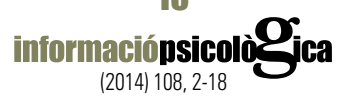


entre los empleados, resultarían estrategias de prevención e intervención que parecen no necesitar de más investigación para gestionar su impacto en la salud mental de estos trabajadores.

Es importante notar que la importancia de las variables parece tener un efecto diferencial en la salud según el país y contexto cultural, por lo que los diagnósticos y las estrategias de intervención deberán considerarlo. El alcance de la presente investigación impide explicar por qué las demandas psicológicas afectan más la salud mental de los profesionales de la salud mexicanos, mientras que el control lo hace en los argentinos o el apoyo social en colombianos y venezolanos. Esta interrogante necesita de otros estudios que analicen cuidadosamente cuestiones culturales que pueden influir en estas relaciones particulares y el desempeño psicométrico de las escalas.

Algunas limitaciones del estudio deben señalarse: su alcance transversal o transeccional, la heterogeneidad en las muestras de cada país, y algunos problemas en la consistencia interna (errores de medición) de las escalas. No obstante lo anterior, en lo que refiere a la heterogeneidad de las muestras y los problemas de consistencia interna, la eliminación de algunos ítems problemáticos así como las correlaciones desatenuadas por error de medición permitieron reducir el impacto de error de medición proporcional a la magnitud en que se presentaba en cada país. Esta estrategia fue considerada necesaria pues el monto de variabilidad por error no fue similar entre los países, y seguramente el peso de las potenciales fuentes de error tampoco. Dejar sin controlar este efecto, al menos desde un enfoque estadístico, aseguraba que los resultados no estarían encubiertos por este problema, al menos desde un enfoque teórico. La influencia más bien errática de las variables demográficas podría originarse de aspectos socioculturales únicos en cada país, asociados no sólo por la idiosincrasia nacional de las personas sino también por aspectos legales y macrosistémicos, aunque no hay en la literatura disponible una tendencia clara de estas variables demográficas en la salud mental. El efecto final de la reducción del impacto error de medición mediante los procedimientos estadísticos aplicados fue que las relaciones tuvieron sentido teórico y práctico, alineándose con la literatura internacional sobre el papel del modelo demanda/control y la salud mental.

Los hallazgos de este estudio en términos de la fuerza y significancia de las variables del modelo demanda/control y la salud mental coinciden con otros estudios en países de desarrollados (Stansfeld y Candy, 2000; Der Doef y Maes, 1999), pero también los realizados en Latinoamérica (Araujo et al, 2003; Pinho y Araujo, 2007; Gómez, 2011; y Urbanetto, et.al 2013). No obstante lo anterior, además de las relaciones diferenciales encontradas entre las variables psicosociales y la salud mental entre países mencionadas antes, también parece haber una discrepancia en el funcionamiento psicométrico de la escala, pues en el presente estudio varios ítems resultaron problemáticos por su contribución al error de medición, aunque en las investigaciones antes citadas, poco se menciona a ese respecto. En una revisión y comparación sobre el funcionamiento de los ítems del JCQ en Latinoamérica (González et. al, 2014), la mayoría de ítems problemáticos varían entre países y estudios, siendo sólo consistentes en su contribución al error (en al menos 7 estudios) dos ítems: el ítem 2 de Control ("mi trabajo implica muchas acciones repetitivas") y el ítem 14 de Demandas

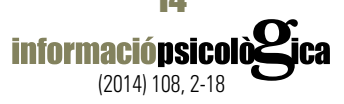


Psicológicas ("tengo suficiente tiempo para terminar mi trabajo), mismos que coinciden plenamente en esta investigación en cada una de las muestras de los países participantes.

Las limitaciones del presente estudio pueden ser el origen de nuevas sugerencias investigativas, por lo que resalta la necesidad de estudios futuros con diseños longitudinales que permitan confirmar con mayor precisión el alcance, magnitud y claridad de las influencias recíprocas que la salud mental y las variables Demandas Psicológicas, Control y Apoyo Social en el trabajo en diferentes ocupaciones y sectores en variados países latinos. Asimismo, parecen indispensables estudios psicométricos del JCQ con mayor profundidad en distintas poblaciones, que incluyan no sólo los análisis estructurales y de consistencia interna, sino de funcionamiento diferencial y de invariancia de escalas e ítems entre distintas poblaciones y sus respectivas exploraciones cualitativas, lo que permitirá concluir con mayor certeza sobre la eficacia general del JCQ independientemente de grupos o culturas.

\section{Agradecimientos}

Agradecemos a todas las personas que contribuyeron en la recolección de datos y sus aportes a esta investigación:

Peter Schnall, PhD, UCI, Paulino Ramírez Osorio, Psic., UNAM, México; Sandra Erín Ramírez Rangel, MPH UNAM, México; Cinthya Flores, UAEM México; Jose Jacinto Ubillus, Lima, Perú, Hiliana Valdivia Romero, Lic. U.Wiener. Lima, Perú; Haydee Raquel Longhi, Lic en Enf., Nuevo Hospital Río Cuarto, Córdova Argentina; Carlo Canepa, Santiago de Chile, y todos aquellos estudiantes y profesores que directa o indirectamente apoyaron en la recolección de datos.

Parte de esta investigación e intercambio entre investigadores fue financiado por el Consejo Nacional de Ciencia y Tecnología (CONACYT) por los proyectos $\mathrm{N}^{\circ} 106068$ and PROINNVOVA N ${ }^{\circ}$ 178161. Agradecemos al programa UCLA Fogarty y el Centro de Epidemiología Social de California por su apoyo en el desarrollo de estos temas en Latinoamérica desde el 2006.

Agradecemos a todos los profesionales de la salud que participaron en el estudio y las autoridades que permitieron la realización del mismo.

\section{Referencias}

Akker, V. M., Buntinx F., Metsemakers J. \& Knottnerus A. (2000). Marginal impact of psychosocial factors on multimorbidity: results of an explorative nested case-control study. Social Science and Medicine, 50, 1679-1693.

Araújo, T. M., Aquino, E., Menezes, G., Oliveira, S. C., \& Aguiar, L. (2003). Aspectos psicossociais do trabalho e distúrbios psíquicos entre trabalhadoras de enfermagem. Revista de Saúde Pública, 37(4), 424-433.

Bourbonnais, R., Comeau, M., \& Vezina, M. (1999). Job strain and evolution of mental health among nurses. Journal of occupational health psychology, 4(2), 95-107.

Courville, T. \& Thompson, B. (2001). Use of structure coefficients in published multiple regression articles: is not enough. Educational and Psychological Measurement, 61, 229-248. 
Cronbach, L. J. (1951).Coefficient alpha and the internal structure of tests. Psychometrika 16, 297-334.

Cronbach, L. J. (2004). My current thoughts on coefficient alpha and successor procedures. Educational and Psychological Measurement, 64, 391-418.

De Jonge, J., \& Kompier, M. A. J. (1997). A critical examination of the Demand-Control-Support Model from a work psychological perspective. International Journal of Stress Management, 4, 235-258.

Der Doef, M. \& Maes, S. (1999). The job demand-control (-support) model and psychological well-being: a review of 20 years of empirical research. Work \& Stress 13,87-114.

Deveraux, J., Buckle P., Peter, W., \& Vlachonicolis I. (1999). Interactions between physical and psychosocial risk factors at work increase the risk of back disorders: an epidemiological approach. Occupational and Environmental Medicine, 56(5), 343-353.

Engström, T., Hanse, J., \& Kadefors, R. (1999). Musculoskeletal symptoms due to technical preconditions in long cycle time work in an automobile assembly plant: a study of prevalence and relation to psychosocial factors and physical exposure. Applied Ergonomics, 30, 443-453.

Estryn-Behar, M., Kaminski, M., Peigne, E., Bonnet, N., V aichère, E., Gozlan, C., Azoulay, S. \& Giorgi, M. (1990). Stress at work and mental health status among female hospital workers. British Journal of Industrial Medicine, 47, 20-28.

Geco, P., Bitencourt T; Magnago, T., Bosi de Souza. L; Prochnow, A; Beck, C., Colomé \& Tavares, J. (2011). Utilização do modelo demanda-controle de Karasek na américa latina: uma pesquisa bibliográfica. Revista de enfermagem da UFSM, 1(2), 272-281.

Gil-Monte, P.R.,\& Peiró, J.M. (1996). Un estudio sobre antecedentes significativos del Síndrome de quemarse por el trabajo" (burnout) en trabajadores de centros ocupacionales para discapacitados psíquicos. Psicología del trabajo y las Organizaciones, 12(1), 67-80.

Goldberg, D., Gater, R., Sartorious, N., Ustun, T. B.., Piccinelli, M. Gureje, O., \& Rutter, C. (1997). The validity of two versions of the GHQ in the WHO study of mental illness in general health care. Psychological Medicine, 27(1), 191-197.

Gómez, V. (2011). Assessment of psychosocial stressors at work: psychometric properties of the JCQ in Colombian workers. Revista Latinoamericana de Psicología, 43(2), 329-342.

González, N; Juárez, A; Camacho, A; Noriega, L \& Escobedo J. (2014). Validez factorial y confiabilidad del cuestionario del contenido del trabajo (JCQ: Job Content Questionnaire) en empleados del Sector Salud, México. En: Juárez-García A. (Coord) (2014). Investigaciones Psicométricas de Escalas Psicosociales en Trabajadores Mexicanos. Plaza y Valdéz: México. En prensa.

Hurrel, J., Murphy, L., Sauter, S., \& Levi, L. (1998). Mental Health. In: Encyclopedia of Occupational Health and Safety (pp 34.6). Sweden: Organización Internacional del Trabajo.

Johnson, J. V. (1986). The impact of workplace and social support, Job demands and work control upon cardiovascular disease (Tesis doctoral, Department of Psychology, University of Stockholm). Sweden Unpublished.

Johnson, J. V., \& Hall, E. M. (1988). Job Strain, Work Place Social Support, and Cardiovascular Disease: A CrossSectional Study of a Random Sample of the Swedish Working Population Amencan Journal of Public Health, 78, 1336-1342.

Johnson, J. W. (2000). A heuristic method for estimating the relative weight of predictor variables in multiple regression. Multivariate Behavioral Research, 35, 1-19.

Johnson, J. W. (2004). Factors affecting relative weights: The influence of sampling and measurement error. Organizational Research Methods, 7, 283-299.

Karasek, R. (1976). The impact of the work environment on life outside the job (Tesis doctoral) Massachusetts Institute of Technology, Cambridge, Massachussetts.

Karasek, R. (1998). Demand/control model: a social, emotional and physiological approach to stress risk and active behavior development. En: Encyclopedia of Occupational Health and Safety (pp 34.6). Sweden; Organización Internacional del Trabajo.

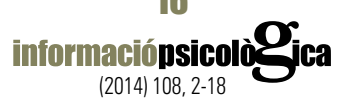


Karasek, R. A. (1979). Job demands, job decision latitude, and mental strain: Implications for job redesign. Administrative Science Quarterly, 24, 285-308.

Karasek, R., \& Theorell, T. (1990). Healthy Work: Stress, Productivity, and the reconstruction of Working Life. USA: Basic Books.

Karasek, R., Brisson, C., Kawakami, N., Houtman, I., Bongers, P., \& Amick, B. (1998). The Job Content Questionnaire (JCQ): An instrument for internationally comparative assessments of psychosocial job characteristics. Journal of Occupational Health Psychology3, 322-355.

Kivimaki, M., Virtanen, M., Elovainio, M., Kouvonen, A., Vaananen, A., \& Vahtera, J. (2006). Work stress in the etiology of coronary heart disease--a meta-analysis. Scand J Work Environ Health, 32(6), 431-442.

Landsbergis, P. A., Schnall, P. L., Warren, K., Pickering, T. G., \& Schwartz, J. E. (1994). Association between ambulatory blood pressure and alternative formulations of job strain. Scand J Work Environ Health, 20(5), 349363.

Lorenzo-Seva, U., Ferrando, P. J., \& Chico, E. (2010). Two SPSS programs for interpreting multiple regression results. Behavior Research Methods, 42, 29-35.

Medina-Mora, E., Padilla C., Campillo, C., Campillo, C., Caraveo, E. \& Corona, J. (1983). The factor structure of the GHQ: a scaled version for hospital's general practice service in México. Psychological Medicine. 13; 355-361.

Michie, S., \& Cockcroft, A. (1996). Overwork can kill - Especially if combined with high demand, low control, and poor social support. British Medical Journal, 312(7036), 921-922.

Niedhammer, I., Bugel, M., , Goldberg, M., Marcel L. \& Guéguen, A. (1998). Psychosocial factors at work and sickness absence in the Gazel Cohort: a prospective study. Occupational and Environmental Medicine, 55(11), 735-741.

Nimon, K., Zientek, R. L. \& Henson, R. K. (2012). The assumption of a reliable instrument and other pitfalls to avoid when considering the reliability of data. Frontiers of Psychology, 3, 102. Disponible en: http://journal.frontiersin.org/Journal/10.3389/fpsyg.2012.00102/full.

Nunnally, J. C., \& Bernstein, I. J. (1995). Teoría psicométrica (3ra. ed.). México, D. F: McGraw-Hill.

Nyberg, S. T., Fransson, E. I., Heikkila, K., Ahola, K., Alfredsson, L., Bjorner, J. B.Kivimaki, M. (2014). Job strain as a risk factor for type 2 diabetes: a pooled analysis of 124,808 men and women. Diabetes Care, 37(8), 22682275. doi: 10.2337/dc13-2936.

Organización Internacional del Trabajo, (2012). Panorama Laboral 2012. América Latina y el Caribe (ILO). Recuperado de http://www.ilo.org/wcmsp5/groups/public/---americas/---ro-lima/documents/publication/ wcms 195884.pdf

Petterson, L. I., \& Arnetz, B. (1997). Meausuring psychosocial work quality and health: Development of health care measures of measurement. Journal of Occupational Health Psychology. 2(3), 229-241.

Pinho, P. S., Araújo, T. M. (2007). Trabalho de Enfermagem em uma unidade hospitalar e transtornos mentais. Rev Enferm UERJ, 15(3), 329-336.

Prady, S. L., Miles, J. N. V., Pickett, K. E., Fairley, L., Bloor, K., Gilbody, S., Kiernan, K., Mann, R. \& Wright, J. (2013). The psychometric properties of the subscales of the GHQ-28 in a multi-ethnic maternal sample: results from the Born in Bradford cohort. BMC Psychiatry, 13(55). Disponible en: http://www.biomedcentral.com/1471244X/13/55

Rodríguez, P., Mendoza, A. (2007). Sistemas productivos y organización del trabajo: Una visión desde Latinoamérica. Gaceta Laboral,13(2),218-241.

Sauter, S. L., Murphy, L. R., \& Hurrell, J. J. (1990). Prevention of work-related psychological disorders. American Psychologist, 45, 1146-1158.

Schnall, L. P., Belkic, K., Landsbergris, P., \& Baker, D. (2000). The Workplace and Cardiovascular Disease. Occupational Medicine, 15, 1-6.

Skov, T., Vilhelm, B., \& Orhede, E. (1996). Psychosocial and physical risk factors for musculoskeletal disorders of the neck, shoulders, and lower back in salespeople. Occupational and Environmental Medicine, 53(5); 351-356. 
Soderfeldt, B., Soderfeldt, M., Muntaner, C., O'Campo, P., Warg, L. E., \& Ohlson, C. G. (1996). Psychosocial work environment in human service organizations: a conceptual analysis and development of the demand-control model. Social Science and Medicine 42(9), 1217-1226.

Spearman, C. (1904).The proof and measurement of association between two things. The American Journal of Psychology, 15, 72-101.

Stansfeld, S. \& Candy, B. (2006). Psychosocial work environment and mental health--a meta-analytic review. Scand J Work Environ Health, 32(6), 443-462.

Theorell, T., Schüldt C., Ekholm, J., \& Michélsen, H. (1995). Physical strength and endurance in relation to perceived psychosocial work environment, sleep disturbance and coping strategies in men. Stockholm MUSIC I (Musculo-Skeletal Intervention Centre) Study Group, Stockholm. Scandinavian Journal of Rehabilitation Medicine, 27(2), 67-71.

Thompson, B. \& Vacha-Haase, T.(2000). Psychometrics is datametrics: the test is not reliable. Educational and Psychological Measurement, 60, 174-195.

Urbanetto, J. d. S., Magalhaes, M. C. C., Maciel, V. O., Sant'Anna, V. M., Gustavo, A. d. S., Poli-de-Figueiredo, C. E., \& Magnago, T. S. B. d. S. (2013). Work-related stress according to the demand-control model and minor psychic disorders in nursing workers. Revista da Escola de Enfermagem da USP, 47, 1180-1186.

Werneke, U., Goldberg, D. P., Yalcin,I. \& Üstun, B. T. (2000). The stability of the factor structure of the General Health Questionnaire. Psychological Medicine, 30(4), 823-829. 